\title{
Livedoid vasculopathy and pathogenesis on the example of a case report
}

\section{Piotr Brzeziński', Jerzy Lasek ${ }^{1}$, Cesar Bimbi², Katarzyna Borowska ${ }^{3}$}

${ }^{1}$ Department of Physiotherapy and Medical Emergency, Faculty of Health Sciences, Pomeranian Academy, Slupsk, Poland, ${ }^{2}$ Brazilian Society of Dermatology, Porto Alegre, Brazil, ${ }^{3}$ Department of Histology and Embryology with Experimental Cytology Unit, Medical University of Lublin, Lublin, Poland

Corresponding author: Piotr Brzeziński, MD PhD, E-mail: brzezoo77@yahoo.com

\begin{abstract}
Livedoid vasculopathy is a painful, ulcerative condition of the lower extremities for which no established treatment exists. The pathogenesis is yet to be understood with the main mechanism being hypercoagulability and inflammation playing a secondary role. The important procoagulant factors include protein $\mathrm{C}$ and $\mathrm{S}$ deficiency, factor V Leiden mutation, antithrombin III deficiency, prothrombin gene mutation and hyperhomocysteinemia. We present a case of a 49 years old woman with dorsoplantar chronic and painful ulcerations known with the disease.
\end{abstract}

Key words: Anticoagulant; Livedoid vasculopathy; Plasminogen activator inhibitor 1; Vascular diseaseslongterm outcome

\section{INTRODUCTION}

Livedoid vasculopathy $(\mathrm{LV})$, also known as atrophie blanche, is associated with chronic venous insufficiency. The clinical presentation of LV consists of multiple, recurrent, painful, punched out ulcers on the lower legs that heal as white, atrophic, stellate scars. The epidermis may be atrophic, ulcerated, or necrotic, with associated dermal sclerosis [1]. These lesions are located particularly around the malleoli. It is 3 times more common in females than in males, especially in patients aged 15 to 50 years [2].

Management involves identifying the lesion and differentiating it from other lower limb lesions along with skin biopsy to confirm the diagnosis. There is no definite first-line treatment, but general measures like smoking cessation, wound care, and pharmacological measures like anticoagulants and antiplatelets have shown good results. Several newer and experimental therapies have shown promising results in resistant cases.
The treatment is multipronged with anti-thrombotic measures such as anti-platelet drugs, systemic anticoagulants and fibrinolytic therapy taking precedence over anti-inflammatory agents. Colchicine, hydroxychloroquine, vasodilators, intravenous immunoglobulin, folic acid, immunosuppressive therapy and supportive measures are also of some benefit.

\section{CASE REPORT}

We present a case of a 49 years old woman with dorsoplantar chronic and painful ulcerations known with the disease. These lesions were partially refractory to systemic therapies and also to local ones.

The treatment history was short and lasted about 2 years.

The patient was previously consulted by dermatologists, vascularsurgeons, internists.

She had no history of smoking or of thrombosis and no varicous visible veins.

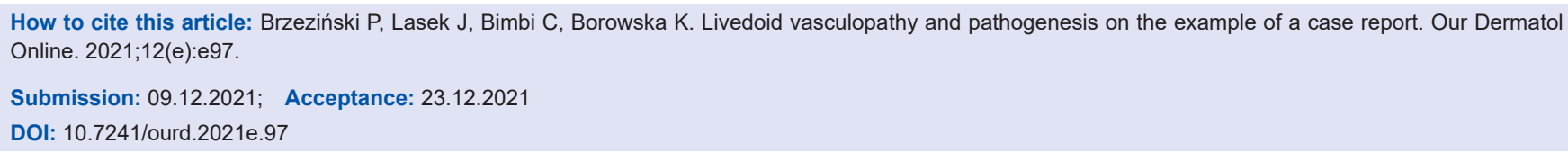




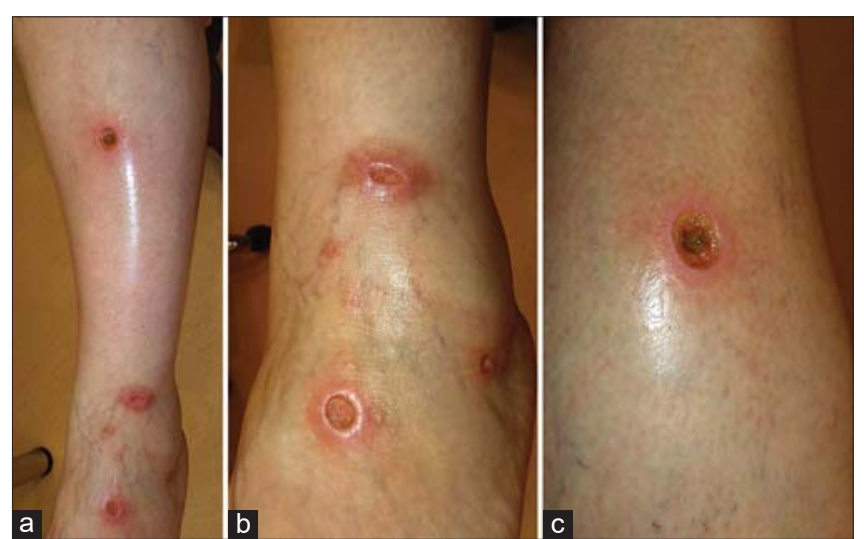

Figure 1: (a-c) Livedoid vasculopathy lesions in a 49-year-old woman on her right leg.

Physical examination revealed small painful ulceration on dorsoplantar area of the left foot and left calf, with some edema and circular erythema around (Figs. la-c). Wound cultures from both ulcers showed Klebsiella with maximum sensitivity to Cefixime. Venous ultrasound was normal.

Anticoagulants were recommended in therapy. In topical therapy silver sulfathiazole, and multiingredient blend with Peruvian balm.

\section{DISCUSSION}

Livedovasculopathy is a disease with livedo racemosa, recurrent ulcers and white scars, which in all probability is based on a procoagulatory coagulation disorder (vasculopathy), although not always definable up to now $[1,3]$.

The elucidation of the pathogenesis of livedovasculopathy down to the molecular level is still the subject of current research efforts. Nevertheless, it is certain that the disease is occlusive with consecutive ischemia of the cutaneous microcirculation [4,5]. The thrombotic occlusions are located in the vessels of the upper and middle dermis, which provide the basis for the cutaneous microcirculation. This then leads to cutaneous ischemia and infarction. In a large number of patients, after intensified diagnostics, abnormal coagulation parameters can accordingly also be detected, which promote excessive thrombosis. To prothrombotic markers with a livedovasculopathy include molecules that directly increase the willingness to clot (e.g. protein $\mathrm{C}$ deficiency) and also reduce physiological thrombolysis (PAI-1 polymorphism) and protein S deficiency, factor V Leiden mutation, antithrombin III deficiency, prothrombin gene mutation and hyperhomocysteinemia.
The largest study to date in this context was carried out at the Mayo Clinic, with the coagulation parameters of 45 patients being screened retrospectively [6]. Prothrombotic abnormalities were recorded in $42 \%$ of the cases, with anticardiolipin antibodies being the most frequently detected. It should be noted that the search for prothrombotic markers can also be unsuccessful, and in view of the cost of the analysis, it is suggested that laboratory diagnostics be carried out in cooperation with a specialized center. The diagnostic challenge is that the prothrombotic markers alone do not allow the diagnosis of lived vasculopathy and are only valid in a clinical context. With regard to microthrombosis valid the Virchow triad of: (a) change in blood composition; (b) change in the vascular wall; (c) change in flow behavior also applies to lived vasculopathy.

The combination of perfusion pressure and temperature in the area of the lower extremities seems to be of particular importance, since the disease manifests itself only in this region, but the blood composition is of course the same systemically. An explanatory model proves that there is a lower concentration of thrombolytic factors in the tissue of the lower extremity and that this results in an increased tendency to thrombosis $[4,7]$. With regard to the associated risk factors for live vasculopathy, it should be noted that individual trigger factors must also occur, since only a small proportion of patients with e.g. B. Protein $\mathrm{C}$ deficiency experiences manifest livedovasculopathy. Lipoprotein $[\mathrm{LP}(\mathrm{a})]$ is an independent risk factor for cardiovascular events. Due to its structural homology to plasmin, Lp (a) inhibits fibrinolysis and thus increases the cutaneous infarct risk by inhibiting the body's own thrombolysis.

\section{CONCLUSION}

- In clinical practice, vasculitis-or skin lesions interpreted as vasculitis-is a constant challenge to the clinician; it often remains underdiagnosed or is diagnosed late.

- The diagnosis is made from the clinical symptoms, the clear anamnesis and the result of the biopsy.

- It is advisable to carry out the extensive coagulation diagnostics in cooperation with specialized coagulation clinics.

- Even if it has not yet been possible to break down all the steps leading to the pathogenesis, classification as a vasculopathy helps to justify anticoagulant therapy. 
- Livedovasculopathy as a chronic recurrent skin infarction places high demands on the diagnostic and therapeutic skills of the treating dermatologist.

\section{Consent}

The examination of the patient was conducted according to the principles of the Declaration of Helsinki.

The authors certify that they have obtained all appropriate patient consent forms, in which the patients gave their consent for images and other clinical information to be included in the journal. The patients understand that their names and initials will not be published and due effort will be made to conceal their identity, but that anonymity cannot be guaranteed.

\section{REFERENCES}

1. Tatu AL. The use of a topical compound cream product with chitosan, silver sulfadiazine bentonite hidrogel and lactic acid for the treatment of a patient with rosacea and ulcerated livedoid vasculopathy. Our Dermatol Online. 2015;6:456-9.

2. Chang D, Patel RM. Livedoid vasculopathy. Cutis. 2012;90:179, 181-2.

3. Tatu AL. Traumatized cervical linear verrucous epidermal nevi - clinical, dermoscopic and treatment challenges. Our Dermatol Online. 2017;8:e10.

4. Goerge T. [Livedoid vasculopathy. Pathogenesis, diagnosis and treatment of cutaneous infarction]. Hautarzt. 2011;62:627-34.

5. Vijayakumar S, Alekhya P, Sasikala M, Ramchandra D. A preliminary study on clinical outcome of corticosteroid therapy in pemphigus patients. Our Dermatol Online. 2014;5:245-50.

6. Callen JP. Livedoid vasculopathy: what it is and how the patient should be evaluated and treated. Arch Dermatol. 2006;142:1481-2.

7. Freitas TQ, Halpern I, Criado PR. Livedoid vasculopathy: a compelling diagnosis. Autops Case Rep. 2018;8:e2018034.

Copyright by Piotr Brzeziński, et al. This is an open access article distributed under the terms of the Creative Commons Attribution License, which permits unrestricted use, distribution, and reproduction in any medium, provided the original author and source are credited.

Source of Support: Nil, Conflict of Interest: None declared. 